\title{
Unlocking Transition for Modulated Surfaces and Quantum Hall Stripes
}

\author{
H.A. Fertig \\ Department of Physics and Astronomy, University of Kentucky, Lexington, KY 40506-0055
}

\begin{abstract}
We develop a sine-Gordon model of layered systems of two-dimensional modulated surfaces and one dimensional stripes, and demonstrate that these systems can undergo a Kosterlitz-Thouless transition in which the modulations unlock as a result of thermal or quantum fluctuations, respectively. The unlocked phase is interpreted as an anisotropic crystal in which soliton-antisoliton pairs proliferate. The properties of such a state for modulated stripes in quantum Hall systems and its possible relevance to recent anomalies in transport data are discussed.
\end{abstract}

Systems which may be modeled as interacting elastic surfaces occur in many different contexts in nature, including liquid crystals, domain walls in magnets [1], electron gases in semiconductor superlattices [2], layered superconductors [3], and biophysical systems [4]. In many situations at a mean-field level the surfaces themselves may contain spatially periodic structure as illustrated in Fig. 1. At very low temperatures, such modulations on different sheets should be strongly correlated so that the system forms a type of three dimensional crystal. An interesting question one may ask is: if the modulations of the surfaces are weakly coupled, can thermal fluctuations cause the modulations on different sheets to become uncorrelated - i.e., unlocked - even if thermal fluctuations do not disorder the modulations within a given surface [5]? In a precise analogy [6], one can also ask whether a series of modulated elastic lines will remain locked at zero temperature when quantum fluctuations are taken into account. It is this latter problem that motivates this work and will be our principle focus.

The modulated elastic line problem is motivated by the theoretical discovery of striped electronic phases in quantum Hall systems in high Landau levels [7 9]. HartreeFock studies of such striped phases at zero temperature 10,11 indicate that they are generically unstable to the formation of modulations along the stripes, so that at the mean-field level the stripe state is essentially an electron (Wigner) crystal [12], albeit a highly anisotropic one. Recent interest in this system has grown due to the discovery [13, 14] of strong anisotropies in the transport properties of high quality two-dimensional electron systems in perpendicular magnetic fields, between quantum Hall plateaus corresponding to filling factors $\nu>4$. $\left(\nu \equiv 2 \pi \rho_{0} l_{0}^{2}, \rho_{0}\right.$ is the electron density, $l_{0}^{2}=\hbar c / e B$ and $B$ is the magnetic field.) In these experiments, dc transport data at very low temperature exhibit a dissipative linear conductivity that is much greater in one direction than the other (i.e., $\sigma_{x x} \gg \sigma_{y y}$ for some direction $x$ [15]). As a function of filling factor, $\sigma_{x x}$ exhibits a strong peak around $\nu_{x}=1 / 2$, where $\nu_{x}$ is the fractional part of the filling factor, while $\sigma_{y y}$ has a minimum around $\nu_{x}=1 / 2$. The system also exhibits unusual non-linear transport: for large applied currents in the direction of high con- ductivity, the the dissipation is greater than what would be expected either from linear response or from a simple heating model [13]. We will argue below that these properties may be understood in the framework of a modulated stripe phase, unlocked by quantum fluctuations 16.

We begin by defining a simple model of sheets with modulations present in them (see Fig. 1). The Hamiltonian may be written as $H=H_{0}+H_{\lambda}$, with

$$
\begin{gathered}
H_{0}=\frac{1}{2} \sum_{j} \int d x d z\left\{\kappa_{x}\left|\partial_{x} u_{j}^{x}\right|^{2}+\kappa_{z}\left|\partial_{z} u_{j}^{x}\right|^{2}\right\} \\
+\frac{1}{2} U \sum_{j} \int d x d z\left[u_{j}^{y}-u_{j-1}^{y}\right]^{2}, \\
H_{\lambda}=-\lambda \sum_{j} \int d x d z \cos \left[\frac{2 \pi}{b}\left(u_{j}^{x}(x, z)-u_{j-1}^{x}(x, z)\right)\right] .
\end{gathered}
$$

The fields $u_{j}^{\mu}(x, z)$ represent displacements of the $j$ th layer in the direction $\mu$ and are the dynamical variables for this problem, $\kappa_{\mu}$ are elastic constants for a given layer, and $U$ represents the energy scale of a harmonicallyapproximated repulsion between layers. In $H_{\lambda}$, the $b$ inside the cosine represents the period of the layer modulations. Since $b$ is the natural length scale in this problem, we will set it to 1 and adopt it as our unit of length [17].

To develop some intuition as to what can happen in this model, it is convenient to "freeze" all the sheets except one; i.e., set $u_{j}^{\mu}=0$ except for $j=0$. If one computes the partition function $Z=\int \mathcal{D} \vec{u}_{0} e^{-H}$ it is easy to see that this separates into a product of two functional integrals, one for each direction of displacement. Of the two integrals, only the one over $u_{0}^{x}$ has a nontrivial structure; it is precisely the partition function for the two-dimensional sine-Gordon model. For small $\lambda$ it is well-known that this model has a Kosterlitz-Thouless phase transition [1]. For large values of $\kappa_{x}$, the $j=0$ sheet will be locked into what we have approximated as the periodic potential of the other (for the moment) static sheets. This state has a set of interesting excitations in 
the form of solitons. At any temperature one should expect to see occasional soliton-antisoliton pairs in the system, but such soliton pairs should always recombine. For the dynamical $(j=0)$ layer, such an excitation represents a finite size patch that has slipped by one period of the modulation (b) relative to the other layers. As $\kappa_{x}$ is decreased, the soliton-antisoliton pairs become more tenuously bound, and the sizes of the patches become increasingly large. At a critical value of $\kappa_{x}$, the solitons fully unbind, leading to arbitrarily large slips of the dynamical surface, and hence its unlocking from the modulations of the others.

In the $2+1$ dimensional quantum problem of modulated lines, the soliton-antisoliton pairs have a simple interpretation: they are vacancy-interstitial pairs forming on a given line. Vacancies and interstitials in a Wigner crystal can carry current 12; thus, if quantum fluctuations populate the ground state with unbound pairs, we expect the system to behave as a metal rather than an insulator. This will be discussed in more detail below.

To properly treat the unlocking transition, we need to include the dynamics of all the layers. A convenient way to approach this is to treat $H_{\lambda}$ as a perturbation, and compute the change in the effective elastic constants $\kappa_{x}$ and $\kappa_{z}$ due to its presence. Following a standard procedure [1], we define the effective stiffnesses of the system by imposing a gradient in the displacement field: $u_{j}^{x}(x, z)=u_{j}^{x(0)}(x, z)+\sum_{q_{y}} e^{i q_{y} j a} \vec{v}_{q_{y}} \cdot \vec{x}$ where $\vec{x}=(x, z)$, $\vec{v}_{q_{y}}=\vec{v}_{-q_{y}}^{*}, a$ is the distance between layers, and the field $u_{j}^{x(0)}(\vec{x})$ must vanish at the system boundaries. The free energy of the system should then take the form $F(v)-$ $F(0)=\frac{\Omega}{2} \sum_{q_{y}} \sum_{\mu, \nu} \kappa_{\mu, \nu}^{R}\left(q_{y}\right) v_{q_{y}}^{\mu} v_{-q_{y}}^{\nu}$ where $\kappa_{\mu, \nu}^{R}\left(q_{y}\right)$ is an effective elasticity tensor, $\Omega$ is the area of a sheet in the $x, z$ plane, and $\mu, \nu=x, z$. Note the $q_{y}$ dependence tells us that the introduction of $H_{\lambda}$ couples displacements in different layers together, even if they are not fully locked.

Using the above expression for the free energy, the renormalized coupling constants may be computed 18 to $O\left(\lambda^{2}\right)$. To this order the resulting elastic constants are diagonal in their indices $\left(\kappa_{\mu, \nu}^{R}=\kappa_{\mu}^{R} \delta_{\mu, \nu}\right)$, and have the form

$$
\kappa_{\mu}^{R}\left(q_{y}\right)=\kappa_{\mu}+\frac{(2 \pi \lambda)^{2}}{4 \Omega} \gamma\left(q_{y}\right) W_{\mu} \int_{a_{c}}^{\infty} d r r^{3}\left(\frac{r}{a_{c}}\right)^{-x_{\kappa}}
$$

In the above expression, $\gamma\left(q_{y}\right)=2-2 \cos \left(q_{y} a\right)$, $\left(W_{x}, W_{z}\right)=\int_{0}^{2 \pi} d \theta\left(\cos ^{2} \theta, \sin ^{2} \theta\right) e^{-f_{\theta}}$, with $f_{\theta}=$ $\pi a \int \frac{d q_{y}}{2 \pi} \gamma\left(q_{y}\right) \ln \left[\frac{\kappa_{z}}{\kappa_{x}} \cos ^{2} \theta+\sin ^{2} \theta\right] /\left(\kappa_{x} \kappa_{z}\right)^{1 / 2}, a_{c}$ is an ultraviolet cutoff of order $b$, and

$$
x_{\kappa}=a \int d q_{y} \frac{\gamma\left(q_{y}\right)}{\left(\kappa_{z} \kappa_{x}\right)^{1 / 2}} .
$$

The quantity $x_{\kappa}$ plays a central role in this model. One may see that the renormalized elasticity constants diverge in perturbation theory if $x_{\kappa} \leq 4$. We take this divergence to signal the onset of the locking transition between the layers. The form of Eq. 3 is typical for a system that undergoes a Kosterlitz-Thouless transition

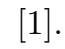

To get an approximation of the phase diagram for this system, it is convenient to expand the elastic constants in a complete set of states, $\kappa_{\mu}\left(q_{y}\right)=\sum_{n} \kappa_{\mu}^{(n)} \gamma_{n}\left(q_{y}\right)$, setting $\gamma_{0}=\left[2-2 \cos \left(q_{y} a\right)\right] / \sqrt{6}$. One can then see that to order $\lambda^{2}$ only $\kappa_{\mu}^{(0)}$ is actually renormalized by the interlayer coupling. To order $\lambda^{2}$ the scaling relations are

$$
\begin{aligned}
\frac{d x_{\kappa}}{d \ell} & =-\lambda^{2} C(\ell) \\
\frac{d \lambda}{d \ell} & =\frac{1}{2}\left[4-x_{\kappa}\right] \lambda
\end{aligned}
$$

with $C(\ell)=\sqrt{6} a \int d q_{y} \gamma_{0}\left(q_{y}\right) \frac{1}{\left(\kappa_{x} \kappa_{z}\right)^{1 / 2}} \sum_{\mu} \frac{W_{\mu}}{\kappa_{\mu}}$. Since $C(\ell)$ behaves smoothly in the vicinity of the critical point, we can set $C(\ell) \rightarrow C(0)$ and only incur errors of order $\lambda^{3}$. With this substitution, one can derive the phase boundary and renormalization group flows in the $\left(x_{\kappa}, \lambda\right)$ plane. The result is illustrated in Fig. 2. It is interesting to note that similar phase diagrams have been obtained in studies of Josephson coupled, layered superconductors [3].

We now turn to the application of this model to the striped phase in the quantum Hall system. Hartree-Fock studies of striped phases [7,8] in high Landau levels indicate that, within mean-field theory, they are unstable to the formation of weak modulations within each stripe 10,11]. The modulation period that is favored turns out to be precisely what is needed so the resulting anisotropic two-dimensional crystal has one electron per unit cell. Qualitatively, the modulations appear to get weaker as the fractional part of the filling factor, $\nu_{x}$, approaches $1 / 2$; however, they never completely vanish, and at $\nu_{x}=1 / 2$ the mean-field state has broken particle-hole symmetry.

To model fluctuations around this mean-field state, we consider each stripe as an elastic line [19], with the modulations coupled by the Hamiltonian $H_{\lambda}$ above. In the absence of modulations, our model potential energy is

$$
\begin{array}{r}
V_{0}=\frac{1}{2} U \sum_{j} \int d x\left[u_{j}^{y}(x)-u_{j-1}^{y}(x)\right]^{2} \\
+\frac{1}{2} \sum_{j} \int d x \kappa_{x}\left(\frac{d u_{j}^{x}(x)}{d x}\right)^{2} .
\end{array}
$$

This model Hamiltonian is most appropriate for electrons interacting via short-range interactions; the effects of long-range interactions will be discussed elsewhere [18]. In the quantum Hall regime, we project the dynamics of the stripes into a single Landau level by making the replacement 20] $u_{j}^{y}(x) \rightarrow \frac{l_{0}^{2}}{i} \frac{\partial}{\partial u_{j}^{x}(x)}$. In the standard fashion [6], one can now compute most quantities of inter- 
est from the generating functional $Z=\int \mathcal{D} u e^{-S}$, with $S=H_{0}+H_{\lambda}$, and [18]

$$
\begin{aligned}
H_{0}=\frac{1}{2} \int d x d \tau \sum_{q_{y}}\left[\kappa_{z}\left(q_{y}\right)\left|\frac{d u\left(x, q_{y}, \tau\right)}{d \tau}\right|^{2}\right. \\
\left.+\kappa_{x}\left|\frac{d u\left(x, q_{y}, \tau\right)}{d x}\right|^{2}\right]
\end{aligned}
$$

where $u\left(x, q_{y}, \tau\right) \equiv \frac{1}{\sqrt{N_{c}}} \sum_{j} e^{-i q_{y} j a} u_{j}^{x}(x, \tau), N_{c}$ is the number of stripes, and $1 / \kappa_{z}\left(q_{y}\right)=U \gamma\left(q_{y}\right) l_{0}^{4}$. $H_{\lambda}$ is formally identical to Eq. 2. Relabeling $\tau \rightarrow z$, except for the $q_{y}$ dependence in the "bare" value of $\kappa_{z}$, the system is formally identical to the one studied above. The analysis follows through essentially without change.

The structure of the phase diagram in Fig. 2 2 suggests that if the stiffnesses of the system $\kappa_{\mu}$ are large enough, or more precisely if $x_{\kappa}$ is small enough, then the system will remain in a locked phase even for arbitrarily small amplitude modulations $\lambda$. Writing $x_{\kappa}$ in terms of the original parameters $\kappa_{x}$ and $U$, one finds that $\left[\frac{\kappa_{x} b^{4}}{U l_{0}^{4}}\right]^{1 / 2}<16 / 3$ to produce an unlocked, free soliton phase. For arbitrarily weak interactions one can estimate the value of this parameter [18]; the result is $\left[\frac{\kappa_{x} b^{4}}{U l_{0}^{4}}\right]^{1 / 2}=\sqrt{2} \pi<16 / 3$. Thus, for weak interactions, we expect the modulations to unlock. We note that it is not immediately obvious what happens as repulsive interactions are turned up: these increase both $\kappa_{x}$ and $U$, and whether their ratio is increased or decreased depends upon the microscopic details of the interaction. Studies of this are currently underway.

In direct analogy with the unpinned phase of the sineGordon equation, the unlocked phase may be thought of as a highly anisotropic Wigner crystal state, in which free solitons - vacancies and interstitials of the Wigner crystal - are included in the ground state due to quantum fluctuations. If the disorder is weak enough, or the temperature high enough, so that localization of the solitons may be ignored [21], these solitons can carry current through the system. The properties of the unlocked phase of this anisotropic Wigner crystal turn out to be consistent with many aspects of the experimental data. (1) Charge transport through the system would be highly anisotropic. Clearly, the solitons are far more mobile along the stripes than across them. Transport across the stripes requires tunneling of the solitons, whose amplitude should be small since the stripe modulations in the mean-field state are weak 222]. (2) In experiment, a peak in the longitudinal conductivity is observed for some direction of transport (e.g., in $\sigma_{x x}$ ) around $\nu_{x}=1 / 2$. If one assumes that the parameters $x_{\kappa}$ and $\lambda$ follow a trajectory as a function of $\nu_{x}$ such as that shown in Fig. 2, then one moves more deeply into the unlocked phase as $\nu_{x} \rightarrow 1 / 2$. The number of carriers - solitons - then would increase as one approaches $1 / 2$. Due to an approximate particle-hole symmetry in the microscopic Hamiltonian [10], this leads to a peak in $\sigma_{x x}$ if the stripes lie along the $x$-direction. A tantalizing possibility for this system is that it can undergo a continuous quantum phase transition as a function of $\nu_{x}$ if the microscopic parameters $x_{\kappa}$ and $\lambda$ pass through the phase boundary (bold line in Fig. (2) as a function of $\nu_{x}$. We note, however, that mean-field calculations [7] support a scenario in which the specific sample studied in Ref. 13 has a first order transition directly into an (unlocked) striped phase. (3) In experiment, a dip is observed in $\sigma_{y y}$ around $\nu_{x}=1 / 2$. In the unlocked phase, linear transport perpendicular to the stripes depends both on the density of solitons and the amplitude for soliton tunneling across stripes. Since the modulations of the stripes become more well-developed as one moves away from $\nu_{x}=1 / 2$ [10], this amplitude should be an increasing function of $\left|\nu_{x}-1 / 2\right|$ [22]. If the amplitude changes fast enough, one would expect to see a dip in $\sigma_{y y}$. A microscopic study of the soliton tunneling amplitude is currently underway. (4) Large currents (in the same direction as that of a measurement of $\sigma_{x x}$ ) lead to anomalously large dissipation in experiment. For the unlocked stripe phase, such a large current would be accompanied by a large electric field parallel to the stripes [18]. Such a field would generate soliton-antisoliton pairs, leading to the enhanced dissipation.

Finally, we comment briefly on other related models currently in the literature. In Refs. [7, 8] striped phases were predicted but the instability to modulations in mean-field theory were not noted. In Ref. 16] the instability is pointed out, shape fluctuations are proposed as a mechanism to restore the stripe phase, and any further effects of modulations on the stripes are not considered. In all these models, tunneling between stripes at zero temperature is not possible due to symmetry considerations, leaving open the question of why $\sigma_{y y}$ is nonvanishing in experiment. Presumably, disorder lifts the symmetry and allows interstripe transport in such models, but to explain the experiments one must adopt a disorder model that allows interstripe tunneling but not localization effects at the lowest experimentally available temperatures. By contrast, in the model discussed here the energy scale for tunneling is set by electron-electron interactions, so for weak disorder it is natural for the system to behave metallically over a range of temperatures. We note also that an interesting possible route to interstripe tunneling, discussed in Ref. [16], is via dislocations if they proliferate (leading to a nematic phase) due to either thermal or quantum fluctuations. Whether the fluctuations in experiment are strong enough to melt the stripe phase in this way is currently unknown; but if present they should lead to interstripe transport in parallel to that discussed in this work. Finally, when described in terms of free vacancies and interstitials the present model admits a simple explanation for the nonlin- 
ear transport properties seen in experiment. It is unclear at present how unmodulated stripes might yield such behavior.

Many interesting issues remain to be explored. Prominent among these are the effect of long-range interactions on the transition, the behavior of the striped quantum Hall phase at finite temperature, the role of edges, the effects of dislocations, and the effects of disorder. These issues are currently under study.

The author thanks many colleagues for useful discussions and comments, including Luis Brey, Sankar Das Sarma, Jim Eisenstein, Charles Hanna, and Allan MacDonald. The author also thanks the ITP at UC Santa Barbara for its hospitality. This work was supported by NSF Grant Nos. DMR98-70681 and PHY94-07194, and by the Research Corporation.

[1] P. M. Chaikin and T.C. Lubensky, Principles of Condensed Matter Physics (Cambridge University Press, New York, 1995).

[2] S. Das Sarma and J.J. Quinn, Phys. Rev. B 25, 7603 (1982).

[3] B. Horovitz, Phys. Rev B 47, 5947 (1993).

[4] A.W.C. Lau, Dov Levine, and P. Pincus, preprint, and references therein.

[5] Layeres of DNA galleries represent a classical realization of such systems for which unlocking transitions have recently been predicted; see L. Golubović and M. Golubović, Phys. Rev. Lett. 80, 4341 (1998); C. O'Hern and T.C. Lubensky, Phys. Rev. Lett. 80, 4345 (1998).

[6] S.L. Sondhi, S.M. Girvin, J.P. Carini, and D. Shahar, Rev. Mod. Phys. 69, 315 (1997).

[7] A.A. Koulakov, M.M. Fogler, and B.I. Shklovskii, Phys. Rev. Lett. 76, 499 (1996).

[8] R. Moessner and J.T. Chalker, Phys. Rev. B 54, 5006 (1996).

[9] For an introduction to the quantum Hall effect, see R.M. Prange and S.M. Girvin, The Quantum Hall Effect, (Springer-Verlag, New York, 1990)..

[10] H.A. Fertig, unpublished.

[11] A.H. MacDonald and M.P.A. Fisher, unpublished.

[12] For a review of two-dimensional Wigner crystals in magnetic fields, see Perspectives in Quantum Hall Effects, S. Das Sarma and A. Pinczuk, eds. (Wiley, New York, 1997), Chaps. 3 and 9.

[13] M.P. Lilly, K.B. Cooper, J.P. Eisenstein, L.N. Pfeiffer, and K.W. West, cond-mat/9808227.

[14] R.R. Du, D.C. Tsui, H.L. Stormer, L.N. Pfeiffer, K.W. Baldwin, and K.W. West, cond-mat/9812025.

[15] At present it is unclear what picks out the direction of larger conductivity in the experiments. In this work we assume some weak underlying structure in the interface at which the electron gas is located picks out a preferred direction for the stripes.
[16] E. Fradkin and S. Kivelson, cond-mat/9810151 have recently suggested that modulated stripes may unlock due to shape fluctuations through a first order transition into a quantum smectic state. The transition discussed in the present work is continuous and presumably different than the one studied by Fradkin and Kivelson.

[17] Dislocations within and between layers are not considered in this model. Their effects will be discussed elsewhere.

[18] Details will be presented elsewhere.

[19] This model ignores tunneling of electrons between stripes.

[20] See, for example, S. Conti and G. Vignale, J. Phys. Cond. Mat. 10, L779 (1998).

[21] Hartree-Fock calculations indicate that the stripe modulations are quite weak, suggesting that the coupling to disorder of the solitons is small.

[22] In the absence of modulations (perfectly uniform stripes), interstripe tunneling vanishes identically due to momentum conservation.

[23] More precisely, the filling factor predicted for the transition from the insulating "bubble phase" of Ref. [0] to the striped phase is very close to the filling factor at which metallic behavior is first observed in Ref. [13].

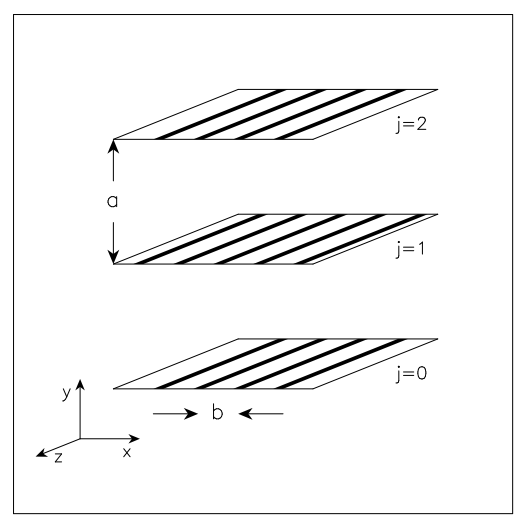

FIG. 1. Example of a modulated sheet system. Shaded areas indicate regions where the two-dimensional sheet density is larger than the average. For repulsive intersheet interactions, at low temperatures one expects the modulations in neighboring sheets to be shifted with respect to one another, forming an anisotropic crystal. 


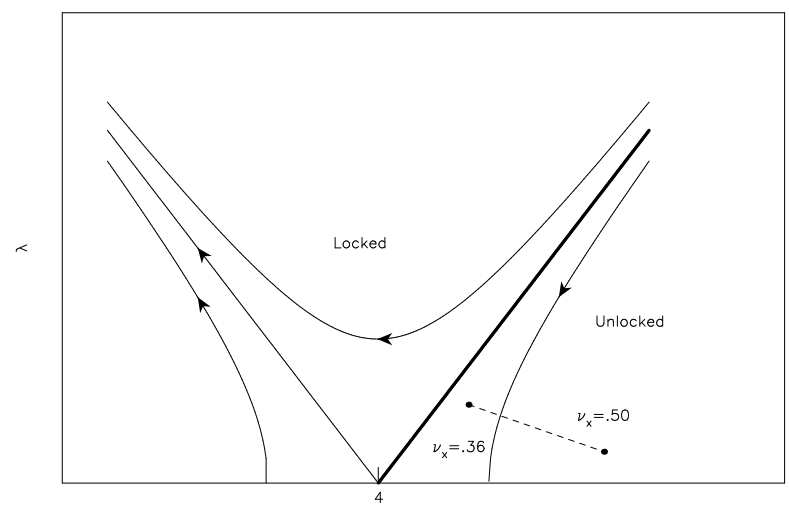

$\mathrm{x}_{\kappa}$

FIG. 2. Phase boundary and renormalization group flows for unlocking transition. Bold line denotes phase boundary. Dotted line illustrates possible initial conditions relevant to the striped phase, discussed in the text. It is assumed (see Ref. 5) that below $\nu_{x} \approx 0.36$ the system is in an insulating "bubble phase" and enters the unlocked stripe phase via a first order transition. Filling factors above 1/2 are related to those below by particle-hole symmetry. 